Enhanced transmission and directivity from metallic subwavelength apertures with nonuniform and nonperiodic grooves

Zhaofeng Li, Humeyra Caglayan, Evrim Colak, and Ekmel Ozbay

Citation: Appl. Phys. Lett. 92, 011128 (2008); doi: 10.1063/1.2829799

View online: http://dx.doi.org/10.1063/1.2829799

View Table of Contents: http://aip.scitation.org/toc/apl/92/1

Published by the American Institute of Physics

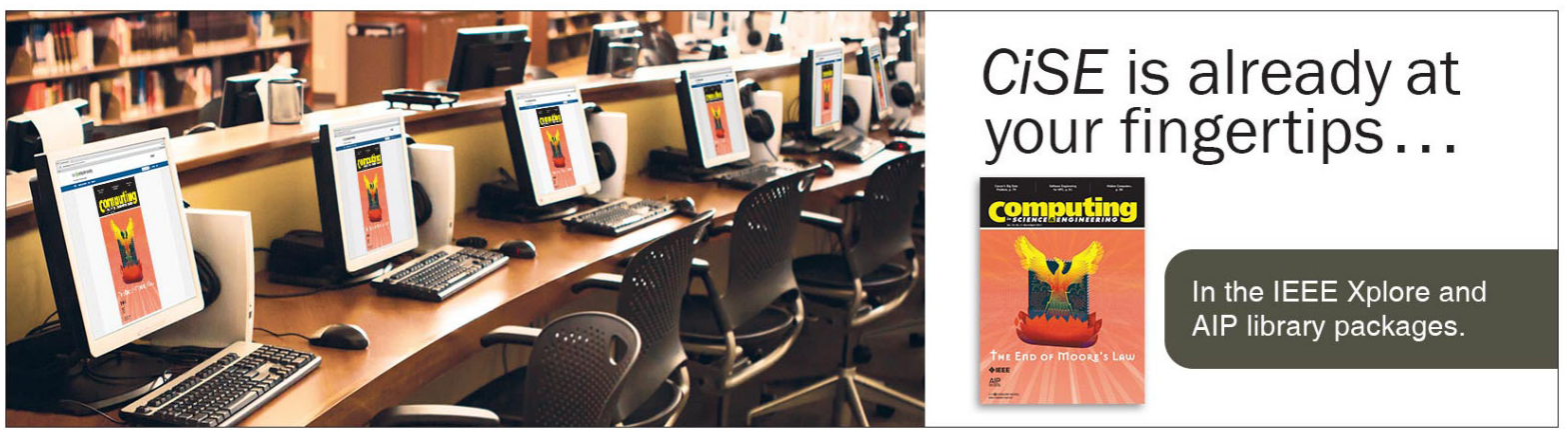




\title{
Enhanced transmission and directivity from metallic subwavelength apertures with nonuniform and nonperiodic grooves
}

\author{
Zhaofeng Li, ${ }^{a}{ }^{2}$ Humeyra Caglayan, Evrim Colak, and Ekmel Ozbay \\ Nanotechnology Research Center, Department of Physics, and Department of Electrical and Electronics \\ Engineering, Bilkent University, Bilkent, 06800 Ankara, Turkey
}

(Received 6 August 2007; accepted 9 December 2007; published online 11 January 2008)

\begin{abstract}
Nonuniform and nonperiodic grooves are used to enhance the transmission and directivity of emissions from a single metallic subwavelength aperture. By using nonuniform and nonperiodic grooves, the amplitude and phase of the diffracted power flow from each groove can be adjusted properly. As a result, the transmission and emission directivity can be further improved when compared to apertures with uniform and periodic grooves. Our experimental results are in good agreement with the finite difference time domain simulation results. (c) 2008 American Institute of Physics. [DOI: 10.1063/1.2829799]
\end{abstract}

Recently, enhanced transmission and beaming light emission from a subwavelength aperture surrounded by periodic corrugations in a metallic film brought about much attention. ${ }^{1,2}$ This phenomenon breaks the limits of homogeneous diffraction and poor transmission through a subwavelength aperture, and might benefit many applications. There were several theoretical and experimental works that discussed the underlying physics and methods to optimize the enhancement of transmission and directional emission. ${ }^{3-5}$ Meanwhile, similar studies were carried out at microwave frequencies. ${ }^{6-11}$ So far, all of the reports on this topic use periodic and uniform grooves, which might be intuitively thought to be the best choice. However, in the present paper, we show that periodic and uniform grooves might not be the best choice for the enhancement transmission and/or directional emission. Instead, by using nonuniform and nonperiodic grooves, the diffracted fields generated from each groove can be adjusted properly, which results in further improved transmission and emission directivity.

Figure 1(a) shows a metal (aluminum) plate (thickness $t=16 \mathrm{~mm}$ ) with a subwavelength aperture (with its width $w=2 \mathrm{~mm}$ ). Arrays of grooves are seen on both sides of the plate. All of the grooves have the same width of $w=2 \mathrm{~mm}$, which is equal to the width of the aperture. This structure is similar to the structure in our previous study. ${ }^{7}$ In Fig. 1(b), there are several parameters that determine the depth and position of the grooves. Since the structures studied here are symmetric about the $x$ and $z$ axes, we only depict a quarter of the structures. $P_{i}(i=1, \ldots, 5)$ is the position of every groove, while $D_{i}$ is the depth. In the present study, a two-dimensional finite difference time domain simulation is carried out to calculate the transmission spectra by using commercial software (FULLWAVE). The space and time steps are set at $\Delta x=\Delta z$. $=0.2 \mathrm{~mm}$, and $c \Delta t=0.1 \mathrm{~mm}$, respectively, where $c$ is the velocity of light in air. Perfectly matched layer boundary conditions are employed to absorb the waves that are reflected from the calculation boundaries. In the simulation, an electromagnetic (EM) plane wave with a $\mathrm{H}$ field polarized in the $y$ direction is incident from the bottom. After the field passes through the aperture, it emits in the $+z$ direction. The transmission spectrum is evaluated by integrating the power flow

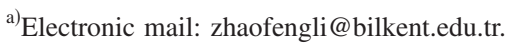

along the cross section of the aperture. Moreover, at a specific frequency, we use six power flow probes (probes $0-5$ ) on the top of the aperture and grooves in order to monitor the amplitude and phase of the power flow contributed by the aperture and each groove. The distance between the probes and grooves is $2 \mathrm{~mm}$.

Now, let us consider a structure with grooves that is uniform and periodic. In this structure, the period of the grooves is set to at $16 \mathrm{~mm}$ (the same period as in our previous study ${ }^{7}$ ). Now, we set out to find the optimal value of the groove depth $D\left(D=D_{1}=D_{2}=D_{3}=D_{4}=D_{5}\right)$, which corresponds to the highest transmission. Figure 2 shows the transmission spectra for structures with different depths, from which it is seen that when $D=3 \mathrm{~mm}$, the transmission spectrum has the highest peak at frequency $f=15.96 \mathrm{GHz}$. Accordingly, we denote this structure with $D=3 \mathrm{~mm}$ as case 1 , and summarize its parameters in Table I. In order to understand the characteristics of the enhanced transmission at $f=15.96 \mathrm{GHz}$, we calculated the amplitudes and phases of the power flows at the $z$ direction that was contributed by the aperture and each groove, the field intensity distribution, and the far field pattern of the emission. Figure 3(a) shows the time-varied power flows that were generated from the aperture and each groove from which we can obtain information of amplitudes and phases. One can see that the power flows vary between positive and negative values, which is the result of the inclusion of vortex waves generated at the surface

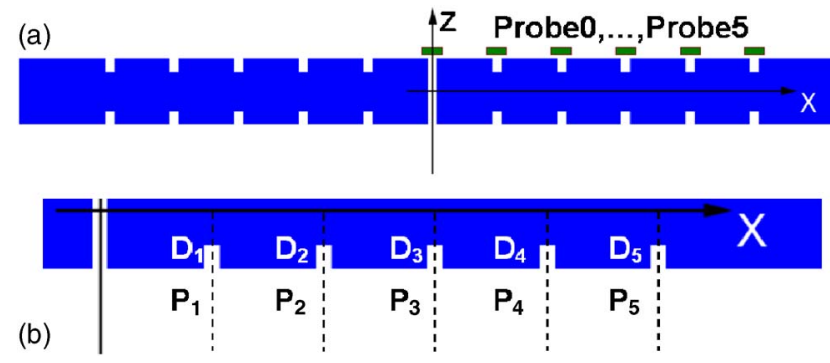

FIG. 1. (Color online) (a) Schematic of a metallic (aluminum) plate with a subwavelength aperture in the middle. On both sides of the plate, there are grooves surrounding the aperture. Above the aperture and grooves, there are six probes to monitor the power flows in the $z$ direction. (b) A quarter of the schematic of (a) which shows the parameters that define the depth and position of the grooves. 


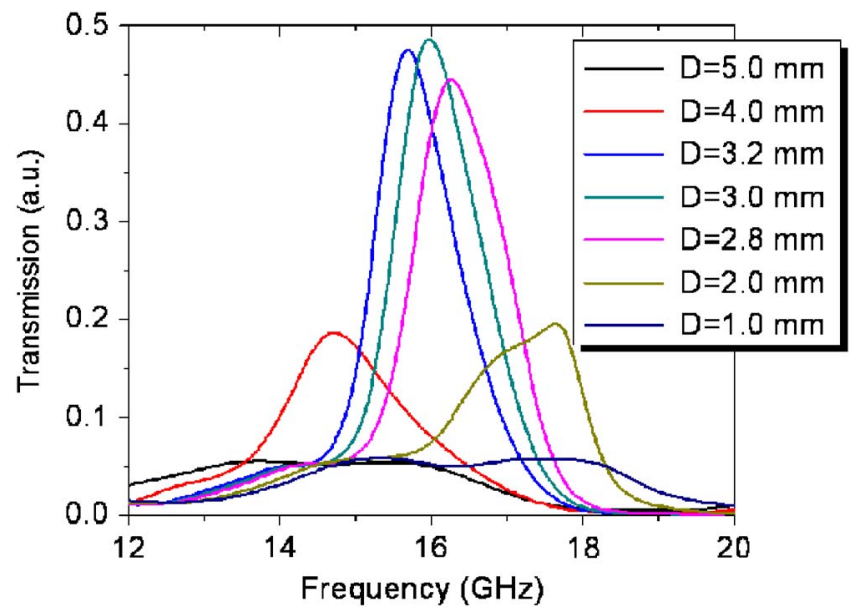

FIG. 2. (Color online) The transmission spectra of case 1 structure with different groove depths.

of the plate. ${ }^{12}$ However, this did not affect our analysis. From Fig. 3(a), it can be seen that, if the groove is located farther away from the aperture, the amplitude of the power flow from the same groove is much lower than that of the grooves closer to the aperture. In addition, the phases of the power flows are different from each other. The phase of the power flow from grooves that are farther away from the aperture is more advanced when compared to the phase of the power flow from grooves that are closer to the aperture. Intuitively, one expects that this phase difference will result in a focus on the emission pattern. Figure 4(a) shows the field intensity distribution of the emission field at $f=15.96 \mathrm{GHz}$. A focus at a distance of approximately $30 \mathrm{~cm}$ can be clearly seen, which verifies our expectation. The far field pattern is shown in Fig. 4(b) with a full width at half maximum (FWHM) of a $9.9^{\circ}$.

Since we have shown that in the structure of case 1, the phases of the power flow generated from the grooves do not match, one can expect an improvement in the transmission and/or directivity of the emission field by designing a structure with uniphase grooves. In order to design such a structure, we adjusted the positions of the grooves based on the structure of case 1 to match the phases of the power flow at each groove for $f=15.96 \mathrm{GHz}$. During the position adjustment, the depth of the grooves were kept constant. We denote this structure as case 2 and summarize its parameters in Table I. Figure 3(b) shows the time-varied power flows generated from the aperture and each groove. When we compare the results of case 2 [Fig. 3(b)] with that of case 1 [Fig. 3(a)], it can be seen that the phases of the power flow match quite well in case 2. So, the introduction of nonperiodic grooves resulted in a power flow that was phase matched at each groove. However, at the same time, the amplitudes of the power flow declined slightly. This is especially true for
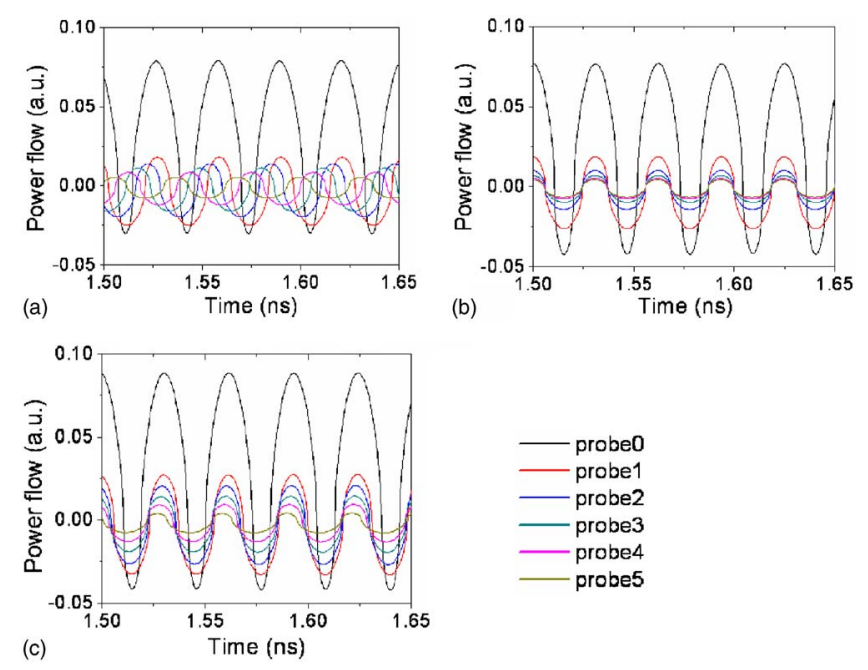

FIG. 3. (Color online) (a), (b), and (c) are the time-varied power flows generated by the aperture and grooves for cases 1, 2, and 3 structures, respectively.

grooves that are farther away from the aperture. The decline of the amplitudes will result in lower transmission, just as the data shown in Table I. In fact, this decline can also be seen in the field intensity distribution plotted in Fig. 4(a) at $f$ $=15.96 \mathrm{GHz}$. Figure 4(b) shows the far field pattern of the emission field. It can be seen that the FWHM reduced to $8.7^{\circ}$ compared to case 1 of $9.9^{\circ}$. Nonetheless, comparing the far field pattern of cases 1 and 2, one finds that the side lobes of case 2 are higher than case 1 . To conclude the structure of case 2, we should say that this structure improved the directivity of the emission field at the cost of transmission efficiency.

We then looked for a structure where we can both improve the transmission and directivity simultaneously. For this purpose, we used nonuniform grooves that have different depths. At a fixed frequency, the grooves with different depths have different diffraction characteristics for an incident EM wave (detailed description will be presented elsewhere). Let us consider the grooves on the incident side of the metal plate. If we set the grooves near the aperture such that they have lower diffraction ability, it is then possible to allow more energy that is diffracted by the grooves away from the aperture to reach the aperture. Conversely, for the emission side, this, in turn, will result in increased power for the EM waves that can reach the grooves away from the aperture, which can be helpful to narrow the emission beam. Based on the above considerations, we adjusted the depth and the position of the grooves. This structure is denoted as case 3 and the parameters are summarized in Table I. Figure 3(c) shows the time-varied power flows generated from the aperture and each groove. For the phases, it is noteworthy that we did not make it fully match, as in case 2 , because we

TABLE I. The structure parameters of three cases and the power transmissions at the peak frequency $f=15.96 \mathrm{GHz}$ of case 1 . Note that the power transmissions are normalized to a value of case 1 .

\begin{tabular}{lccccccccccc}
\hline \hline & $\begin{array}{c}D_{1} \\
(\mathrm{~mm})\end{array}$ & $\begin{array}{c}D_{2} \\
(\mathrm{~mm})\end{array}$ & $\begin{array}{c}D_{3} \\
(\mathrm{~mm})\end{array}$ & $\begin{array}{c}D_{4} \\
(\mathrm{~mm})\end{array}$ & $\begin{array}{c}D_{5} \\
(\mathrm{~mm})\end{array}$ & $\begin{array}{c}P_{1} \\
(\mathrm{~mm})\end{array}$ & $\begin{array}{c}P_{2} \\
(\mathrm{~mm})\end{array}$ & $\begin{array}{c}P_{3} \\
(\mathrm{~mm})\end{array}$ & $\begin{array}{c}P_{4} \\
(\mathrm{~mm})\end{array}$ & $\begin{array}{c}P_{5} \\
(\mathrm{~mm})\end{array}$ & $\begin{array}{c}\text { Transmission at } \\
f=15.96 \mathrm{GHz}\end{array}$ \\
\hline Case 1 & 3.0 & 3.0 & 3.0 & 3.0 & 3.0 & 16 & 32 & 48 & 64 & 80 & 1 \\
Case 2 & 3.0 & 3.0 & 3.0 & 3.0 & 3.0 & 15 & 32.6 & 51 & 69.6 & 88.6 & 0.91 \\
Case 3 & 2.4 & 2.6 & 2.8 & 3.0 & 3.4 & 17 & 33.8 & 51 & 68.6 & 86.2 & 1.23 \\
\hline \hline
\end{tabular}



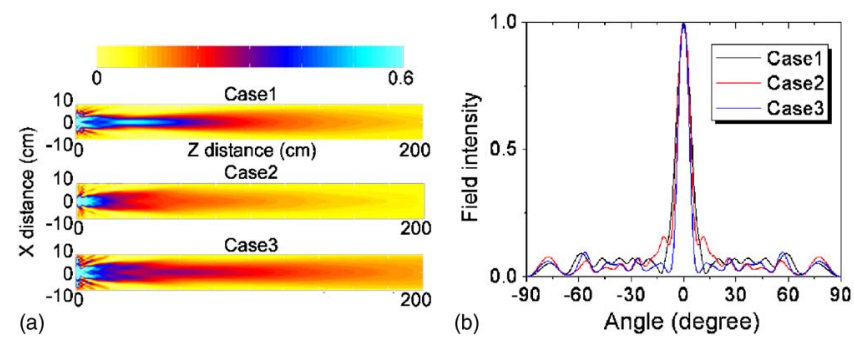

FIG. 4. (Color online) (a) The $H$ field intensity distributions of emissions at frequency of $15.96 \mathrm{GHz}$ for cases 1,2 , and 3 structures, respectively. (b) The far field patterns for the three cases. The FWHMs of cases 1, 2, and 3 are $9.9^{\circ}, 8.7^{\circ}$, and $7.2^{\circ}$, respectively.

made a trade-off between improving the directivity and suppressing the sidelobe in the far field pattern. By comparing Figs. 3(c) and 3(a), it can be seen that most of the amplitudes of the power flow for case 3 are slightly increased when compared to case 1 . This increase of amplitude results in an improved transmission, which is now higher than case 1, as shown by the data in Table I. Figure 4(a) shows the field intensity distribution of the emission field at $f=15.96 \mathrm{GHz}$, which confirms the improvement of the transmission. The far field pattern is plotted in Fig. 4(b) with its FWHM at 7.2 $2^{\circ}$. It can be seen that the sidelobes are suppressed effectively when compared to case 2 . Therefore, we obtained a structure (case 3) that simultaneously has higher transmission and better directivity than that of case 1 .

It is well known that the surface wave is the key factor for funneling EM wave through the subwavelength aperture and beaming the wave from the aperture. ${ }^{3,4}$ It is noteworthy that by using a uniform and periodic groove array, the incoming EM wave can be converted into surface waves more efficient than using a nonperiodic one. However, these surface waves will propagate evenly in two opposite directions. Only the surface wave propagating toward the aperture is helpful to the funneling effect, while the surface wave in the opposite direction is mostly wasted. By using a nonuniform and nonperiodic structures, it is possible to increase the surface wave toward the aperture while suppress the surface wave which is leaving the aperture (detailed data will be presented elsewhere). Consequently, a nonuniform and nonperiodic groove array is possibly more efficient than a uniform and periodic one to enhance transmission through a subwavelength aperture.

To verify the above simulation results, we conducted experiments for the three structures. In the experiment, a HP$8510 \mathrm{C}$ network analyzer was used to excite a horn antenna to obtain an incident EM field. Another horn antenna that was connected to the same network analyzer was used to receive and measure the power emitted from the aperture. Since it is difficult to measure the fields in the aperture, we measure the field intensities along the $z$ axis. Figure 5(a) shows the measured data (discrete one) together with the simulated data (solid lines). One can see that the experimental data agree well with the simulation results. Figure 5(b) shows the measured far field patterns for the three cases. The FWHMs of cases 1,2 , and 3 are $10.3^{\circ}, 9.0^{\circ}$, and $7.3^{\circ}$, respectively. The FWHMs of the measured data are slightly larger than the
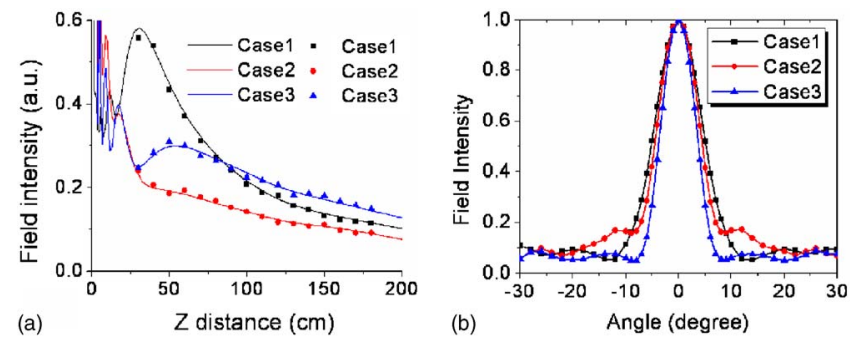

FIG. 5. (Color online) (a) The field intensity along the $z$ axis at a frequency of $15.96 \mathrm{GHz}$. The solid lines are simulation data, while the discrete dots are measured data. The measured data are normalized by setting the value of case 1 at $180 \mathrm{~cm}$ to be equal to the value of simulation. (b) The measured far field patterns of three case structure. The measured FWHMs of cases 1, 2, and 3 are $10.3^{\circ}, 9.0^{\circ}$, and $7.3^{\circ}$, respectively.

calculated results. This might result from the fact that while the structure and the experiments were made in three dimensions, the simulations were carried out on a two-dimensional structure. Additionally, considering other factors such as the manufacturing precision of the samples, the measurement of far field radiation pattern, etc., the experimental patterns are in good agreement with the calculated results shown in Fig. 4(b).

In summary, we demonstrated that a uniform and periodic groove structure might not be the best choice for the enhanced transmission and directional emission from a metallic subwavelength aperture. By using a nonuniform and nonperiodic groove structure, it is possible to adjust the amplitudes and phases of the power flows that are generated from each groove properly. As a result, the transmission and directivity can simultaneously be further improved. Our experimental results confirm our simulation results rather well.

This work was supported by the European Union under the projects EU-NoE-METAMORPHOSE, EU-NoEPHOREMOST, and TUBITAK under Project No. 105E066, 105A005, 106E198, and 106A017. One of the authors (E.O.) also acknowledges partial support from the Turkish Academy of Sciences.

${ }^{1}$ T. Thio, K. M. Pellerin, R. A. Linke, H. J. Lezec, and T. W. Ebbesen, Opt. Lett. 26, 1972 (2001).

${ }^{2}$ H. J. Lezec, A. Degiron, B. Devaux, R. A. Linke, L. Martin-Moreno, F. J. Garcia-Vidal, and T. W. Ebbesen, Science 297, 820 (2002).

${ }^{3}$ L. Martin-Moreno, F. J. Garcia-Vidal, H. J. Lezec, A. Degiron, and T. W. Ebbesen, Phys. Rev. Lett. 90, 167401 (2003).

${ }^{4}$ F. J. Garcia-Vidal, H. J. Lezec, T. W. Ebbesen, and L. Martin-Moreno, Phys. Rev. Lett. 90, 213901 (2003).

${ }^{5}$ L.-B. Yu, D.-Z. Lin, Y.-C. Chen, Y.-C. Chang, K.-T. Huang, J.-W. Liaw, J.-T. Yeh, J.-M. Liu, C.-S. Yeh, and C.-K. Lee, Phys. Rev. B 71, 041405(R) (2005).

${ }^{6}$ M. J. Lockyear, A. P. Hibbins, J. R. Sambles, and C. R. Lawrence, Appl. Phys. Lett. 84, 2040 (2004).

${ }^{7}$ S. Sena Akarca-Biyikli, I. Bulu, and E. Ozbay, Appl. Phys. Lett. 85, 1098 (2004).

${ }^{8}$ S. Sena Akarca-Biyikli, I. Bulu, and E. Ozbay, J. Opt. A, Pure Appl. Opt. 7, S159 (2005).

${ }^{9}$ H. Caglayan, and I. Bulu, and E. Ozbay, Opt. Express 13, 1666 (2005).

${ }^{10}$ H. Caglayan, I. Bulu, and E. Ozbay, J. Opt. Soc. Am. B 23, 419 (2006).

${ }^{11}$ H. Caglayan, I. Bulu, and E. Ozbay, Microwave Opt. Technol. Lett. 48, 2491 (2006).

${ }^{12}$ R. Gordon, Phys. Rev. B 75, 193401 (2007). 